Article

\title{
The Vasorelaxant Mechanisms of a Rho Kinase Inhibitor DL0805 in Rat Thoracic Aorta
}

\author{
Lili Gong ${ }^{1,2}$, Jianhao Peng ${ }^{1}$, Lianhua Fang ${ }^{1, *}$, Ping Xie ${ }^{1}$, Kun Si ${ }^{3}$, Xiaozhen Jiao ${ }^{1}$, \\ Liping Wang ${ }^{1}$ and Guanhua Du ${ }^{1, *}$
}

1 Beijing City Key Laboratory of Drug Target Identification and Drug Screening, State Key Laboratory for Bioactive Substances and Functions of Natural Medicines, Institute of Materia Medica, Chinese Academy of Medical Science \& Peking Union Medical College, Beijing 100050, China; E-Mails: gonglili2010@yahoo.com.cn (L.G.); pengjianhao@hotmail.com (J.P.); xp@imm.ac.cn (P.X.); jiaoxz@imm.ac.cn (X.J.); together3171@hotmail.com (L.W.)

2 Beijing Chao-Yang Hospital affiliated with Beijing Capital Medical University, Beijing 100020, China

3 Department of Macromolecular Science and Engineering, Case Western Reserve University, Cleveland, OH 44106, USA; E-Mail: kun.si1@case.edu

* Authors to whom correspondence should be addressed; E-Mails: fanglh@imm.ac.cn (L.F.); dugh@imm.ac.cn (G.D.); Tel.: +86-10-6316-5184 (G.D.); Fax: +86-10-6301-7757 (G.D.).

Received: 26 March 2012; in revised form: 11 May 2012 / Accepted: 11 May 2012 /

Published: 18 May 2012

\begin{abstract}
Rho-kinase has been suggested as a potential therapeutic target in the treatment of cardiovascular diseases. The Rho-kinase signaling pathway is substantially involved in vascular contraction. The aim of the present study was to evaluate the vasorelaxant effects of Rho kinase inhibitor DL0805 in isolated rat aortic rings and to investigate its possible mechanism(s). It was found that DL0805 exerted vasorelaxation in a dose-dependent manner in $\mathrm{NE}$ or $\mathrm{KCl}$-induced sustained contraction and partial loss of the vasorelaxation under endothelium-denuded rings. The DL0805-induced vasorelaxation was significantly reduced by the nitric oxide synthase inhibitor $\mathrm{N}^{\omega}$-nitro-L-arginine methyl ester, the guanylate cyclase inhibitor methylene blue and the cyclooxygenase inhibitor indomethacin. The voltage-dependent $\mathrm{K}^{+}$channel blocker 4-aminopyridine remarkably attenuated DL0805-induced relaxations. However, the ATP-sensitive $\mathrm{K}^{+}$channel blocker glibenclamide and $\mathrm{Ca}^{2+}$-activated $\mathrm{K}^{+}$channel blocker tetraethylammonium did not affect the DL0805-induced relaxation. In the endothelium-denuded rings, DL0805 also reduced NE-induced transient contraction and inhibited contraction induced by increasing external
\end{abstract}


calcium. These findings suggested that DL0805 is a novel vasorelaxant compound associated with inhibition of Rho/ROCK signaling pathway. The NO-cGMP pathway may be involved in the relaxation of DL0805 in endothelium-intact aorta. The vasorelaxant effect of DL0805 is partially mediated by the opening of the voltage-dependent $\mathrm{K}^{+}$channels.

Keywords: DL0805; vasorelaxation; Rho kinase; aorta; endothelium; nitric oxide

\section{Introduction}

Rho kinase, also known as Rho-associated coiled-coil forming protein kinase (ROCK), is a serine/threonine protein kinase that is the downstream effector of Rho GTPases [1]. The Rho/ROCK pathway is well known as a major regulator of vascular smooth muscle cells (VSMCs) contraction and plays an important role in hypertension, atherosclerosis, and pulmonary hypertension [2,3]. The essential regulatory mechanism of smooth muscle contraction is myosin light chain (MLC) phosphorylation by the $\mathrm{Ca}^{2+}$-calmodulin-activated MLC kinase (MLCK) and dephosphorylation by the $\mathrm{Ca}^{2+}$-independent MLC phosphatase (MLCP) activity [4]. ROCK mediates the MLC phosphorylation by inhibiting the enzyme activity of MLCP [5,6]. Bussemaker et al. demonstrated that ROCK was involved in the regulation of endothelial nitric oxide synthase (NOS) [7,8], while other researchers indicated that NO induces dilation of rat aorta via inhibition of ROCK signaling pathway $[9,10]$. Rho-kinase inhibitors Y-27632 and fasudil have been used as tool compounds to evaluate the role of ROCK proteins in various disease models. Y-27632 [11] and fasudil [12] have been shown to exhibit relaxation effects on vessels.

5-Nitro-1(2) $H$-indazole-3-carbonitrile (DL0805) is a new ROCK inhibitor derived from a compound database through high-throughput screening (HTS) as previously described [13]. DL0805 is a homologue of 7029, a Rho kinase inhibitor which was found in that previous study [13]. When we evaluated the relaxant effects of the lead compounds identified in the HTS DL0805 showed the strongest vasorelaxant effects. Few studies have been carried out regarding the effect of DL0805 on vascular smooth muscle contraction. Thus, the purpose of the present study was to evaluate the relaxant effects of DL0805 in the rat thoracic aorta and its possible mechanism(s).

\section{Results and Discussion}

\subsection{Inhibition Effect of DL0805 on ROCK}

ROCK-I inhibitory activity was observed in the Kinase-Glo Luminesce Kinase Assay High-throughput screening and virtual screening. As shown in Table 1, the $\mathrm{IC}_{50}$ value for DL0805 was determined to be $6.67 \mu \mathrm{M}$. 
Table 1. ROCK-I inhibitors identified by virtual screening and high-throughput screening. Pharmacophore-Fit Value, LigandFit-Dock Score, and $\mathrm{IC}_{50}$ values for DL0805 and Y-27632.

\begin{tabular}{lllll}
\hline Compound ID & $\mathbf{I C}_{\mathbf{5 0}}(\boldsymbol{\mu M})$ & FitValue & DockScore \\
\hline SL0805 & & & \\
\end{tabular}

\subsection{Relaxant Effects of DL0805 on Aortic Rings Contracted by $\mathrm{NE}$ and $\mathrm{KCl}$}

In order to evaluate the effects of DL0805 on the contraction induced by norepinephrine (NE) and $\mathrm{KCl}$, endothelium-intact aortic rings pre-contracted with $\mathrm{NE}$ or $\mathrm{KCl}$ were used. DL0805 relaxed the $\mathrm{NE}(1 \mu \mathrm{M})$ pre-contracted aortic rings in a dose-dependent manner. DL0805 also relaxed aortic rings pre-contracted with $\mathrm{KCl}(60 \mathrm{mM})$ in a similar way (Figure $1 \mathrm{~A})$.

Figure 1. Vasorelaxant effects of DL0805 on endothelium-intact thoracic aorta rings contracted with NE or KCl. (A) Effects of DL0805 on endothelium-intact thoracic aorta rings pre-contracted with $\mathrm{NE}(1 \mu \mathrm{M})$ or $\mathrm{KCl}(60 \mathrm{mM})$. (B) Effects of DL0805 (5, 10, 25, $50 \mu \mathrm{M})$ on $\mathrm{NE}\left(10^{-9} \sim 10^{-6} \mathrm{M}\right)$ and $(\mathbf{C}) \mathrm{KCl}(10 \sim 60 \mathrm{mM})$ induced contraction. Results are presented as means \pm S.E.M., $\mathrm{n}=6 . * P<0.05, * * P<0.01$ compared with vehicle control.
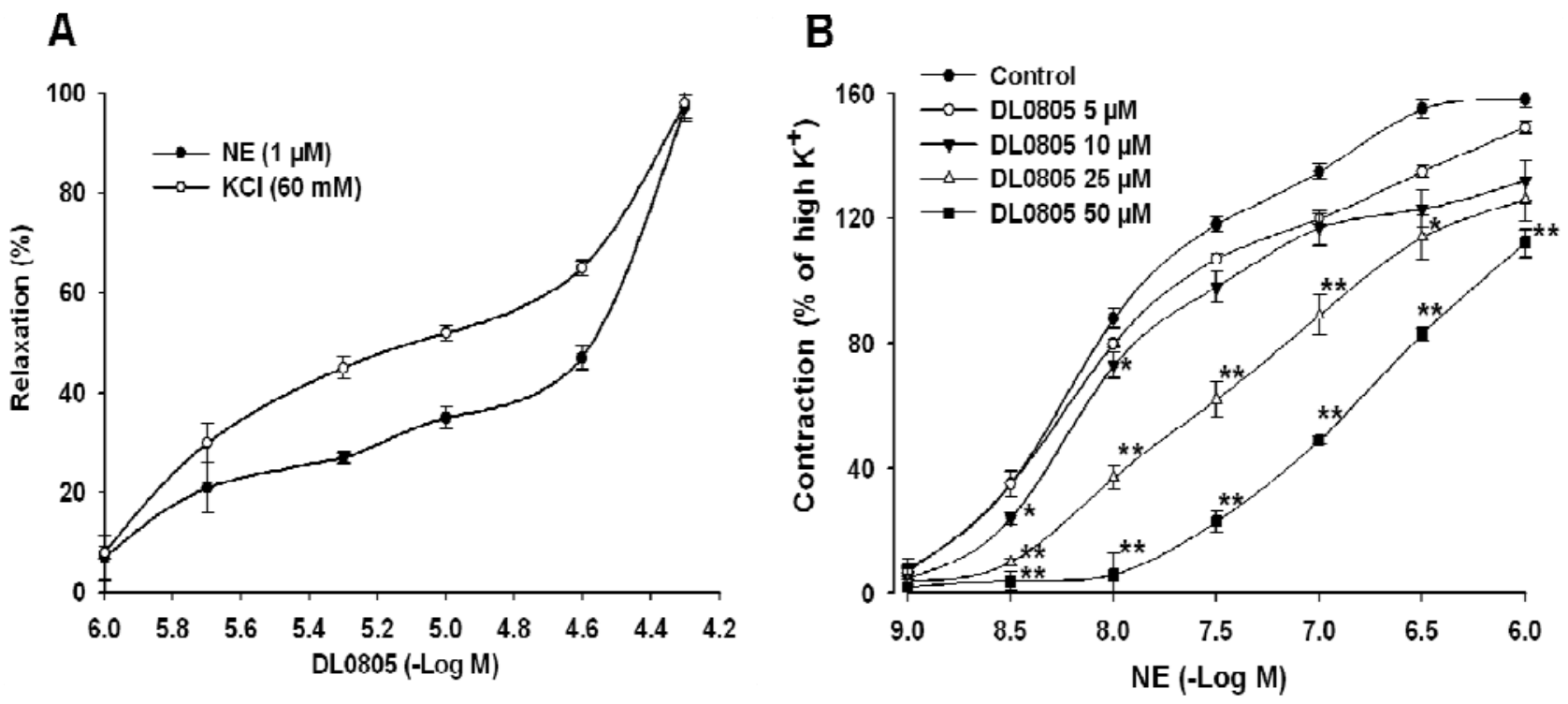
Figure 1. Cont.

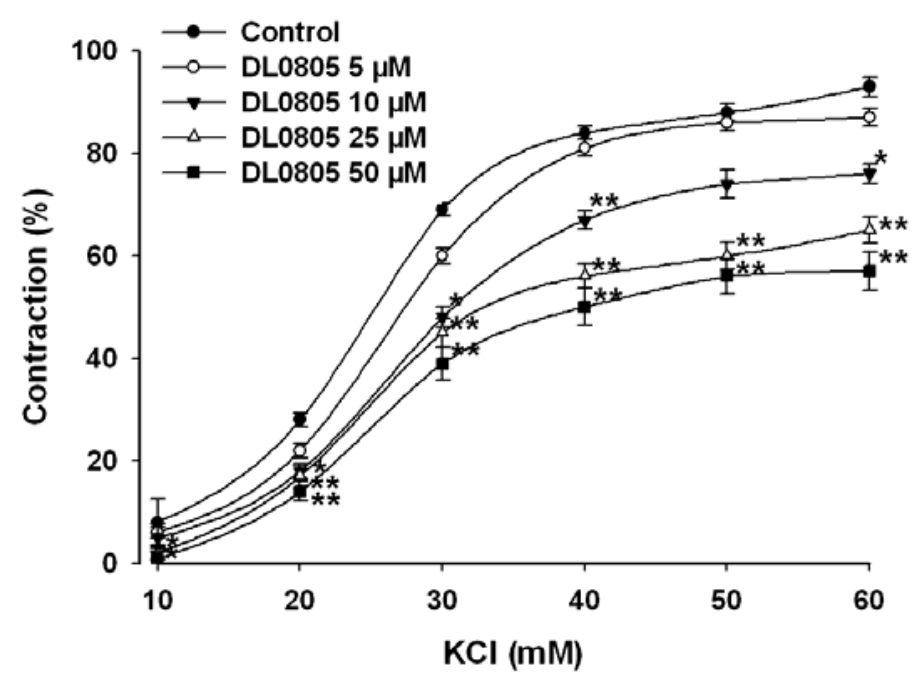

Pre-incubation with DL0805 $(5,10,25$ and $50 \mu \mathrm{M})$ inhibited the concentration response contraction of NE in a parallel fashion, and depressed the maximal responses to $107.0 \pm 4.0 \%, 101.1 \pm 4.5 \%$, $87.3 \pm 4.1 \%$ and $57.3 \pm 2.8 \%$, respectively ( $v s$. vehicle control group $111.0 \pm 2.0 \%, \mathrm{n}=6$ ) (Figure 1B) (pA2 value $4.03 \pm 0.51 ; \mathrm{n}=6$ ). We also observed that 5, 10, 25 and $50 \mu \mathrm{M}$ DL0805 inhibited the contractile response to $\mathrm{KCl}$, and depressed the maximal responses to $87.1 \pm 1.7 \%, 76.5 \pm 1.9 \%$, $65.3 \pm 2.6 \%$ and $57.8 \pm 3.7 \%$, respectively (vs. vehicle group $93.0 \pm 1.9 \%, \mathrm{n}=6$ ) (Figure 1C) ( $\mathrm{pIC}_{50}$ value $3.75 \pm 0.46 ; \mathrm{n}=6$ ).

\subsection{Role of Endothelium in DL0805 Induced Relaxation of Aortic Ring}

To elucidate the role of endothelium in DL0805-mediated vasorelaxation, concentration-response to DL0805 was studied in endothelium-intact and endothelium-denuded rings pre-contracted by NE $(1 \mu \mathrm{M})$. The relaxation effect of DL0805 in endothelium-intact aorta was significantly stronger than that in endothelium-denuded aorta. In endothelium-denuded rings, DL0805 produced a partial relaxation with maximal effect $97.6 \pm 2.6 \%$ ( $v$ s. endothelium-intact group $109.1 \pm 1.8 \%, \mathrm{n}=6$ ) (Figure 2A). Removal of functional endothelium inhibited the relaxant response to DL0805, suggesting that the vasorelaxation caused by DL0805 was endothelium-dependent.

Since DL0805 induced both endothelium-dependent and -independent relaxation in isolated rat aortic rings, an attempt was made to investigate what endothelium-derived vasoactive factors contributed to the DL0805-induced relaxation. Pre-incubation of endothelium-intact rings with the NOS inhibitor $\mathrm{N}^{\omega}$-nitro-L-arginine methyl ester (L-NAME, $100 \mu \mathrm{M}$ ), the guanylate cyclase inhibitor methylene blue $(5 \mu \mathrm{M})$ and the cyclooxygenase inhibitor indomethacin $(5 \mu \mathrm{M})$ for 20 min before NE $(1 \mu \mathrm{M})$ was added, and then DL0805 $(1 \sim 50 \mu \mathrm{M})$ was added. We found that L-NAME, methylene blue and indomethacin significantly reduced the DL0805 induced relaxation, with maximal relaxant effects of $93.3 \pm 1.5 \%, 91.2 \pm 2.4 \%$ and $74.5 \pm 3.2 \%$, respectively ( $v$ s. control group $109.1 \pm 1.8 \%, \mathrm{n}=6$, Figure $2 \mathrm{~B}$ ). These results indicate that the NO-cGMP and endothelium cyclooxygenase pathways may be involved in the relaxation of DL0805 in endothelium-intact aorta. 
Figure 2. Vasorelaxant effects of DL0805 on the contraction induced by NE $(1 \mu \mathrm{M})$ in the aortic rings with (+Endo) or without (-Endo) endothelium (A) and effects of pre-incubation of L-NAME $(100 \mu \mathrm{M})$, methylene blue $(5 \mu \mathrm{M})$ and indomethacin $(5 \mu \mathrm{M})$ on DL0805 induced relaxation in endothelium-intact aorta $(\mathbf{B})$. Results are presented as means \pm S.E.M., $\mathrm{n}=6 . * P<0.05, * * P<0.01$ compared with endothelium-intact aorta $(\mathbf{A})$ or $* P<0.05$, $* * P<0.01$ compared with control $(\mathbf{B})$.

A

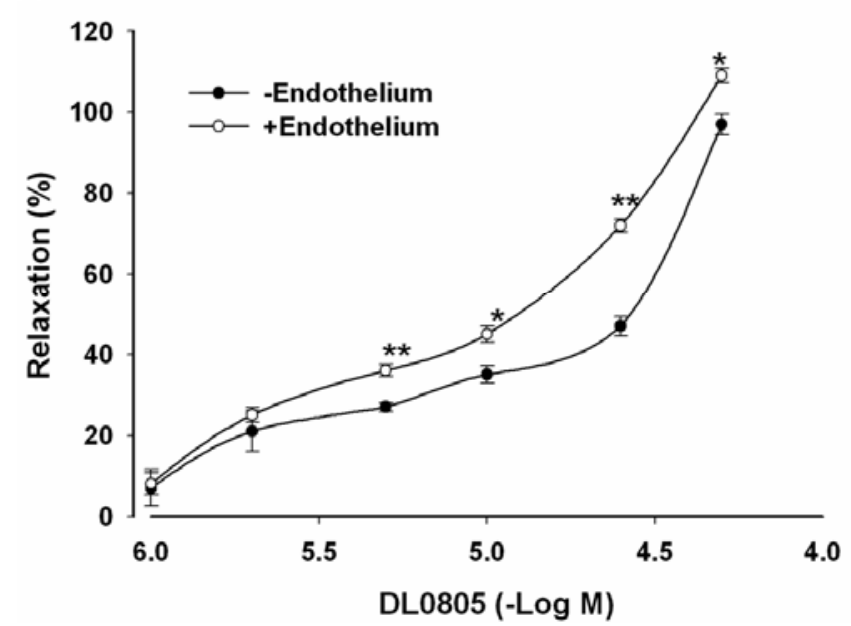

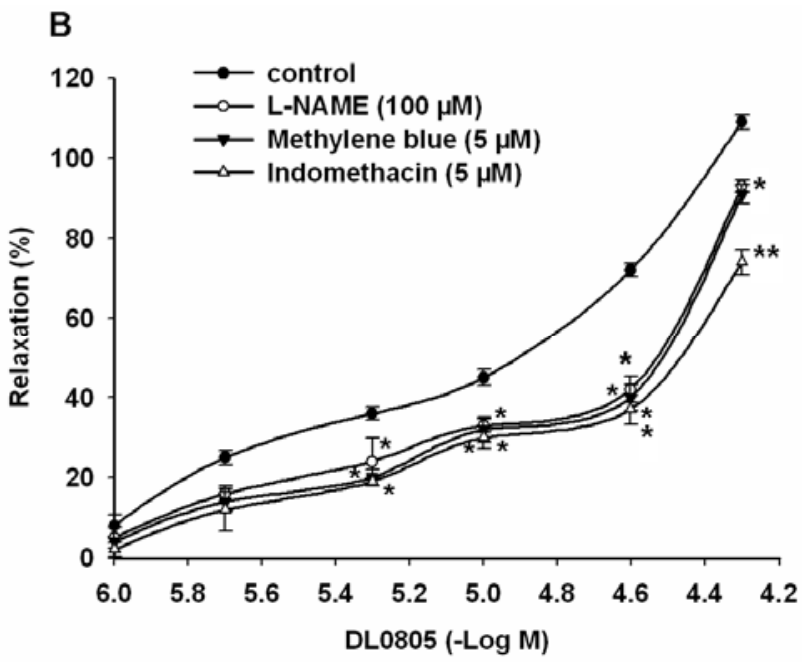

\subsection{Role of $\mathrm{K}^{+}$Channels in DL0805 Induced Relaxation}

$\mathrm{K}^{+}$channels play an important role in the regulation of muscle contractility and vascular tone [14]. There are several types of $\mathrm{K}^{+}$conductance present in vascular smooth muscle and they are subject to modulation by various factors. To demonstrate the role of $\mathrm{K}^{+}$channels in DL0805-induced relaxation, endothelium denuded aortic rings were pre-incubated with $\mathrm{K}^{+}$channel blockers. We used three $\mathrm{K}^{+}$ channel blockers: the ATP-sensitive $\mathrm{K}^{+}$channel (KATP) blocker glibenclamide, the $\mathrm{Ca}^{2+}$-activated $\mathrm{K}^{+}$ channel (KCa) blocker tetraethylammonium (TEA), and the voltage-dependent $\mathrm{K}^{+}$channel $(\mathrm{Kv})$ blocker 4-aminopyridine (4-AP). Pretreatment with 4-AP $(100 \mu \mathrm{M})$ attenuated DL0805-induced relaxations pre-contracted by NE. However, pretreatment with glibenclamide $(10 \mu \mathrm{M})$ and TEA $(5 \mathrm{mM})$ did not significantly affect the DL0805-induced relaxation (Figure 3). It is probable that DL0805 activates Kv channels in rat endothelium-denuded arteries [15]. These results indicate that the vasorelaxant effect of DL0805 is partially mediated by the opening of the $\mathrm{K}^{+}$channels in VSMCs.

\subsection{Effect of DL0805 on Extracellular $\mathrm{Ca}^{2+}$ Induced Contraction and Intracellular $\mathrm{Ca}^{2+}$ Release in Aortic Ring}

The influx of external $\mathrm{Ca}^{2+}$ through specific $\mathrm{Ca}^{2+}$ channels or $\mathrm{Ca}^{2+}$ release from internal stores plays an important role in excitation-contraction coupling of smooth muscle. As we know, there are two kinds of $\mathrm{Ca}^{2+}$ channels in the VSMCs: Voltage-dependent $\mathrm{Ca}^{2+}$ channels and receptor-operated $\mathrm{Ca}^{2+}$ channels [16]. 
Figure 3. Effects of pre-incubation of glibenclamide $(10 \mu \mathrm{M})$, TEA $(5 \mathrm{mM})$ and 4-AP $(100 \mu \mathrm{M})$ on DL0805 induced relaxation in thoracic aorta rings precontracted by NE $(1 \mu \mathrm{M})$. Results are presented as means \pm S.E.M., $\mathrm{n}=6, * P<0.05, * * P<0.01$ compared with control.

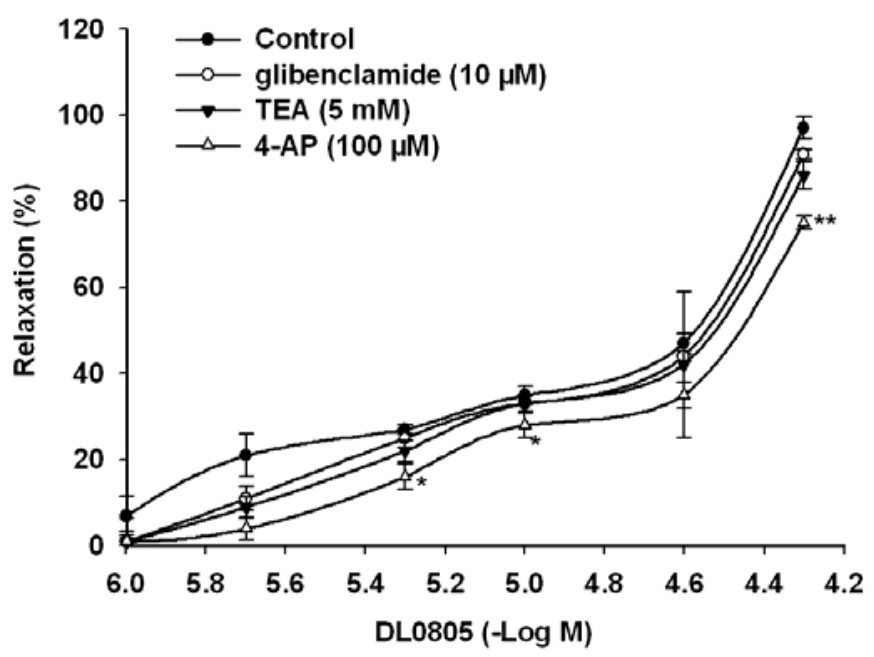

To determine whether the inhibition of extracellular $\mathrm{Ca}^{2+}$ influx is involved in DL0805 induced relaxation, the experiments were carried out in $\mathrm{Ca}^{2+}$-free Krebs-Henseleit $(\mathrm{K}-\mathrm{H})$ solution [17]. The high $\mathrm{K}^{+}$-induced contraction of smooth muscle is the result of an increase in $\mathrm{Ca}^{2+}$ influx through voltage-dependent $\mathrm{Ca}^{2+}$ channels [18]. In the $\mathrm{Ca}^{2+}$-free solution plus $60 \mathrm{mM} \mathrm{KCl}$, cumulative addition of $\mathrm{CaCl}_{2}(0.1 \sim 2.5 \mathrm{mM})$ induced a stepwise tension increase of aortic rings. Pretreatment with 5, 10, 25 and $50 \mu \mathrm{M}$ DL0805 noticeably attenuated $\mathrm{CaCl}_{2}$ induced contraction (Emax $58.1 \pm 4.4 \%, 12.7 \pm 1.3 \%$, $10.9 \pm 1.6 \%$ and $9.2 \pm 1.7 \%$, respectively, $\mathrm{n}=6$ ) (Figure 4A). These results supporting that DL0805 exhibited $\mathrm{Ca}^{2+}$ entry blocking activity.

Figure 4. Inhibitory effect of DL0805 on the cumulative-contraction curve dependent on extracellular $\mathrm{Ca}^{2+}$ influx induced by $\mathrm{KCl}(60 \mathrm{mM})$ in $\mathrm{Ca}^{2+}$-free solution added cumulatively $\mathrm{CaCl}_{2}(0.1 \sim 2.5 \mathrm{mM})(\mathbf{A})$ and on the $\mathrm{NE}(1 \mu \mathrm{M})$ induced contraction in $\mathrm{Ca}^{2+}$-free solution (B). Results are presented as means \pm S.E.M., $\mathrm{n}=6, * P<0.05$, $* * P<0.01$ compared with vehicle.
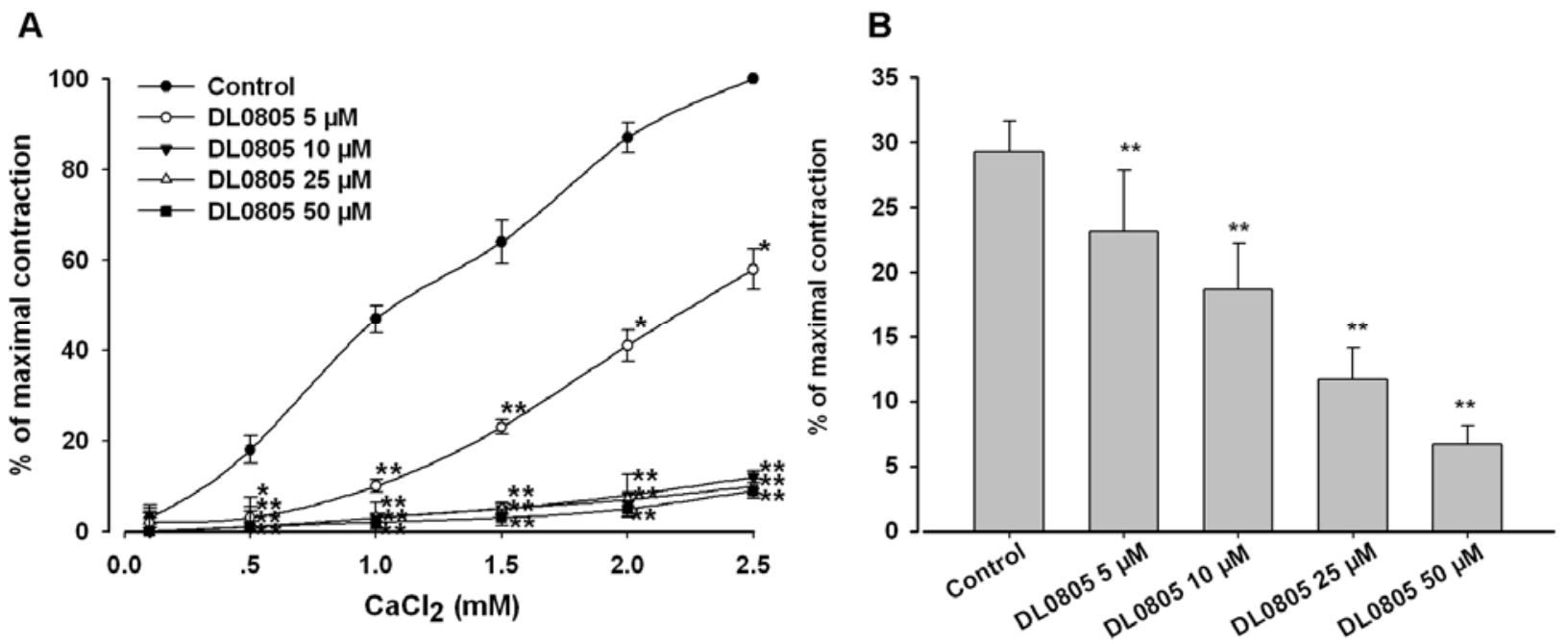
To clarify whether the relaxation induced by DL0805 was related to inhibition of intracellular $\mathrm{Ca}^{2+}$ release, experiments were carried out in $\mathrm{Ca}^{2+}$-free $\mathrm{K}-\mathrm{H}$ solution containing $50 \mu \mathrm{M}$ EGTA [19]. By acting on specific membrane receptors, $\mathrm{NE}$ induces $\mathrm{Ca}^{2+}$ influx through receptor-operated channels causing tonic contraction [20]. In the $\mathrm{Ca}^{2+}$-free solution, $\mathrm{NE}(1 \mu \mathrm{M})$ induced a transient contraction due to the release of intracellular $\mathrm{Ca}^{2+}$. As shown in Figure 4B, pretreatment with DL0805 $(5,10,25$ and $50 \mu \mathrm{M}$ ) significantly reduced the contraction induced by NE, and the Emax was $23.1 \pm 4.7 \%$, $18.8 \pm 3.5 \%, 11.7 \pm 2.5 \%, 6.6 \pm 1.4 \%$, respectively ( $v s$. vehicle $29.3 \pm 2.4 \%, \mathrm{n}=6)$. DL0805 may also inhibit $\mathrm{Ca}^{2+}$ mobilization from intracellular stores. Taken together, these results indicate that DL0805 may be acting as a $\mathrm{Ca}^{2+}$ antagonist.

\section{Experimental}

\subsection{Chemicals and Drugs}

NE, acetylcholine (ACh), indomethacin, L-NAME, methylene blue, glibenclamide, TEA and 4-AP were purchased from Sigma (St. Louis, MO, USA). DL0805 was synthesized by the Department of Medicinal Chemistry of our institute and its structure was confirmed by the analysis physical-chemical properties and spectral evidence.

\subsection{Screening of ROCK InhibitorDL0805}

High-throughput screening and virtual screening for the identification of novel ROCK inhibitors was done as previously described [13]. Simply, we developed common-feature pharmacophore models and LigandFit model to compare the structure and the interaction with ROCK between Y-27632 and DL0805. A new high-throughput screening model based on Kinase-Glo Luminescent Kinase Assay (Promega, Madison, WI, USA) was established to identify the inhibition effect of DL0805 on ROCK.

\subsection{Isometric Tension Measurements}

All studies were approved by the Laboratories Institutional Animal Care and Use Committee of the Chinese Academy of Medical Sciences and Peking Union Medical College. Male Sprague-Dawley rats (250-300 g) were anesthetized with pentobarbitone sodium $(60 \mathrm{mg} / \mathrm{kg}$, i.p.). The thoracic aorta was immediately excised and immersed in ice-cold $\mathrm{K}-\mathrm{H}$ solution with the following composition (mM): $\mathrm{NaCl} 120, \mathrm{KCl} 4.8, \mathrm{KH}_{2} \mathrm{PO}_{4}$ 1.2, $\mathrm{NaHCO}_{3}$ 25, glucose 11, $\mathrm{CaCl}_{2} 2.5, \mathrm{MgCl}_{2} 1.4$ and ethylene-diaminetetraacetic acid (EDTA) 0.01. After removed debris tissue, the aorta was cut into rings of about $2 \sim 3 \mathrm{~mm}$ in length. For endothelium-denuded aorta, endothelium was mechanically removed by gently rubbing the lumen with a wet cotton ball and the absence of acetylcholine-induced relaxation was used as a denuding indicator [21].

The tension of aortic rings was recorded isometrically via a force displacement transducer connected to a BIO-PAC polygraph (MP100A). The aortic rings were hanged in organ baths containing $10 \mathrm{~mL} \mathrm{K-H}$ solution which was maintained at $37{ }^{\circ} \mathrm{C}$ and gassed continuously with a $95 \%$ $\mathrm{O}_{2}$ and 5\% $\mathrm{CO}_{2}$ mixture [22] and equilibrated for $60 \mathrm{~min}$ under a $1.2 \mathrm{~g}$ resting tension. During the equilibration period, K-H solution was changed every $20 \mathrm{~min}$. The aortic rings were given two successive stimulations with high $\mathrm{K}^{+}(60 \mathrm{mM})$ solution, which was prepared by replacing $\mathrm{NaCl}$ with 
equimolar $\mathrm{KCl}$ in $\mathrm{K}-\mathrm{H}$ solution. The tension reached about $3 \mathrm{~g}$ when treated with $60 \mathrm{mM} \mathrm{K}$. The endothelial integrity was confirmed by eliciting a relaxation with ACh $(10 \mu \mathrm{M})$ after contraction induced by $\mathrm{NE}(1 \mu \mathrm{M})$. Only endothelium-intact rings exhibiting more than $60 \%$ relaxation to ACh were used for the experiments [23]. While in endothelium denuded rings, the relaxation to ACh was less than 5\%. Submaximal contraction was induced using NE $(1 \mu \mathrm{M})$, then cumulative dose-response curves to the DL0805 (1 50 $\mu \mathrm{M})$. With inhibitors (L-NAME, methylene blue or indomethacin) or vehicle, the vessels were pretreated with such compounds prior to submaximal contraction with NE then determined the endothelial response to the tested compounds. TEA, glibenclamide and 4-AP were also used to demonstrate the role of $\mathrm{K}^{+}$channels on DL0805 induced relaxation. In order to clarify the $\mathrm{Ca}^{2+}$ release, the experiments were carried out in $\mathrm{Ca}^{2+}$-free $\mathrm{K}-\mathrm{H}$ solution.

\subsection{Statistical Analysis}

All data were expressed as the means \pm S.E.M. Statistical analysis was performed using the one-way ANOVA or Student's t-test. The $P$ value less than 0.05 was regarded as significantly different.

\section{Conclusions}

In conclusion, the results suggest that DL0805 exerts its vasodilatory effects by acting on multiple sites. DL0805 induces relaxation in rat aortic rings through endothelium-dependent and -independent pathways. NO-cGMP mediated pathway may be involved in the endothelium-dependent relaxation. DL0805 also blocks extracellular $\mathrm{Ca}^{2+}$ influx by interacting with both voltage- and receptor-operated $\mathrm{Ca}^{2+}$ channels. The Rho/ROCK pathway has been shown to induce vascular smooth muscle contraction, suppress the expression of eNOS, and increase intracellular $\mathrm{Ca}^{2+}$ concentration. DL0805 act as a novel vasorelaxant compound associated with inhibition of Rho/Rho-kinase signaling pathway.

\section{Acknowledgments}

This study was supported by the National Natural Science Foundation of China (No. 30572182), the special foundation on scientific and technological basic work that is provided by China Ministry of Science and Technology (No. 2007FY130100) and International Scientific and Technological Cooperation Projects of China (No. 2009DFA32010).

\section{References and Notes}

1. Leung, T.; Manser, E.; Tan, L.; Lim, L. A novel serine/threonine kinase binding the Ras-related RhoA GTPase which translocates the kinase to peripheral membranes. J. Biol. Chem. 1995, 270, 29051-29054.

2. Loirand, G.; Guerin, P.; Pacaud, P. Rho kinases in cardiovascular physiology and pathophysiology. Circ. Res. 2006, 98, 322-334.

3. Shimokawa, H.; Takeshita, A. Rho-kinase is an important therapeutic target in cardiovascular medicine. Arterioscler. Thromb. Vasc. Biol. 2005, 25, 1767-1775.

4. de Godoy, M.A.; Rattan, S. Role of rho kinase in the functional and dysfunctional tonic smooth muscles. Trends Pharmacol. Sci. 2011, 32, 384-393. 
5. Chu, J.; Miller, C.T.; Kislitsyna, K.; Laine, G.A.; Stewart, R.H.; Cox, C.S.; Uray, K.S. Decreased myosin phosphatase target subunit 1(MYPT1) phosphorylation via attenuated rho kinase and zipper-interacting kinase activities in edematous intestinal smooth muscle. Neurogastroenterol. Motil. 2012, 24, 257-266, e109.

6. Wilson, D.P.; Susnjar, M.; Kiss, E.; Sutherland, C.; Walsh, M.P. Thromboxane A2-induced contraction of rat caudal arterial smooth muscle involves activation of $\mathrm{Ca}^{2+}$ entry and $\mathrm{Ca}^{2+}$ sensitization: Rho-associated kinase-mediated phosphorylation of MYPT1 at Thr-855, but not Thr-697. Biochem. J. 2005, 389, 763-774.

7. Bussemaker, E.; Pistrosch, F.; Forster, S.; Herbrig, K.; Gross, P.; Passauer, J.; Brandes, R.P. Rho kinase contributes to basal vascular tone in humans: Role of endothelium-derived nitric oxide. Am. J. Physiol. 2007, 293, H541-H547.

8. Liu, C.Q.; Leung, F.P.; Wong, S.L.; Wong, W.T.; Lau, C.W.; Lu, L.; Yao, X.; Yao, T.; Huang, Y. Thromboxane prostanoid receptor activation impairs endothelial nitric oxide-dependent vasorelaxations: The role of Rho kinase. Biochem. Pharmacol. 2009, 78, 374-381.

9. Chitaley, K.; Webb, R.C. Nitric oxide induces dilation of rat aorta via inhibition of rho-kinase signaling. Hypertension 2002, 39, 438-442.

10. Shiga, N.; Hirano, K.; Hirano, M.; Nishimura, J.; Nawata, H.; Kanaide, H. Long-term inhibition of RhoA attenuates vascular contractility by enhancing endothelial NO production in an intact rabbit mesenteric artery. Circ. Res. 2005, 96, 1014-1021.

11. Freitas, M.R.; Eto, M.; Kirkbride, J.A.; Schott, C.; Sassard, J.; Stoclet, J.C. Y27632, a Rho-activated kinase inhibitor, normalizes dysregulation in alpha1-adrenergic receptor-induced contraction of Lyon hypertensive rat artery smooth muscle. Fundam. Clin. Pharmacol. 2009, 23, 169-178.

12. Li, Q.; Chen, Y.; Sun, L.; Fu, G.; Guo, L. Vasodilatation produced by Fasudil Mesylate in vivo and in vitro. Vascul. Pharmacol. 2011, 55, 121-126.

13. Gong, L.L.; Fang, L.H.; Peng, J.H.; Liu, A.L.; Du, G.H. Integration of virtual screening with high-throughput screening for the identification of novel Rho-kinase I inhibitors. J. Biotechnol. 2010, 145, 295-303.

14. Dick, G.M.; Tune, J.D. Role of potassium channels in coronary vasodilation. Exp. Biol. Med. (Maywood) 2010, 235, 10-22.

15. Tang, Q.Y.; Zhang, Z.; Xia, X.M.; Lingle, C.J. Block of mouse Slo1 and Slo3 $\mathrm{K}^{+}$channels by CTX, IbTX, TEA, 4-AP and quinidine. Channels (Austin) 2010, 4, 22-41.

16. Sanders, K.M. Regulation of smooth muscle excitation and contraction. Neurogastroenterol. Motil. 2008, 20 (Suppl. 1), 39-53.

17. Tirapelli, C.R.; Ambrosio, S.R.; da Costa, F.B.; Coutinho, S.T.; de Oliveira, D.C.; de Oliveira, A.M. Analysis of the mechanisms underlying the vasorelaxant action of kaurenoic acid in the isolated rat aorta. Eur. J. Pharmacol. 2004, 492, 233-241.

18. van Breemen, C.; Farinas, B.R.; Gerba, P.; McNaughton, E.D. Excitation-contraction coupling in rabbit aorta studied by the lanthanum method for measuring cellular calcium influx. Circ. Res. 1972, 30, 44-54. 
19. Jiang, H.D.; Cai, J.; Xu, J.H.; Zhou, X.M.; Xia, Q. Endothelium-dependent and direct relaxation induced by ethyl acetate extract from Flos Chrysanthemi in rat thoracic aorta. J. Ethnopharmacol. 2005, 101, 221-226.

20. Paoletti, R.; Govoni, S. Classification of calcium antagonists: proposal of the WHO Committee. Pharmacol. Res. Commun. 1987, 19, 195-208.

21. Brayden, J.E. Membrane hyperpolarization is a mechanism of endothelium-dependent cerebral vasodilation. Am. J. Physiol. 1990, 259, H668-H673.

22. Fang, L.H.; Mu, Y.M.; Lin, L.L.; Xiao, P.G.; Du, G.H. Vasorelaxant effect of euxanthone in the rat thoracic aorta. Vascul. Pharmacol. 2006, 45, 96-101.

23. Zhu, X.M.; Fang, L.H.; Li, Y.J.; Du, G.H. Endothelium-dependent and -independent relaxation induced by pinocembrin in rat aortic rings. Vascul. Pharmacol. 2007, 46, 160-165.

Sample Availability: Not available.

(C) 2012 by the authors; licensee MDPI, Basel, Switzerland. This article is an open access article distributed under the terms and conditions of the Creative Commons Attribution license (http://creativecommons.org/licenses/by/3.0/). 